\title{
ANALYTIC SEMIGROUP APPROACH TO GENERALIZED NAVIER-STOKES FLOWS IN BESOV SPACES
}

\author{
ZHI-MIN CHEN
}

\begin{abstract}
The energy dissipation of the Navier-Stokes equations is controlled by the viscous force defined by the Laplacian $-\Delta$, while that of the generalized Navier-Stokes equations is determined by the fractional Laplacian $(-\Delta)^{\alpha}$. The existence and uniqueness problem is always solvable in a strong dissipation situation in the sense of large $\alpha$ but it becomes complicated when $\alpha$ is decreasing. In this paper, the well-posedness regarding to the unique existence of small time solutions and small initial data solutions is examined in critical homogeneous Besov spaces for $\alpha \geq \frac{1}{2}$. An analytic semigroup approach to the understanding of the generalized Navier-Stokes equations is developed and thus the well-posedness on the equations is examined in a manner different to earlier investigations.
\end{abstract}

Keywords: Generalized Navier-Stokes equations, well-posedness, analytic semigroup, Besov spaces

Mathematics Subject Classification: 35B32, 35B35, 35Q35, 86A10

\section{INTRODUCTION}

The Navier-Stokes equations, governing the motion of incompressible and viscous fluid substances through the balance of viscous force, pressure force and fluid acceleration, are a fundamentally important system in fluid mechanics. The three-dimensional Navier-Stokes equations also give rise to Leray's question of global existence of classical solutions [27]. This is of great interest in mathematical analysis. In the attempt to answer the question, a variety of dissipative equations were examined (see, for example, [13, 22, 25, 31, 32, 36]).

In the present paper, we are interested in the generalized Navier-Stokes equations

$$
\partial_{t} u+(-\Delta)^{\alpha} u+u \cdot \nabla u+\nabla \pi=0, \quad \nabla \cdot u=0
$$

in the domain $(0, T) \times R^{d}$ for the dimension number $d \geq 2$ and the time bound $0<T \leq \infty$. Here $u$ and $\pi$ represent respectively unknown fluid velocity and pressure. The fluid motion described by (1) involves an energy dissipation which is controlled by the fractional Laplacian $(-\Delta)^{\alpha}$. The existence, uniqueness and regularity of the solution to (1) is essentially determined by the parameter $\alpha$. When $\alpha=1$, equation (1) is the Navier-Stokes equations and thus Leray's question remains open for $d \geq 3$. Equation (1) with $\alpha>1$ is called a hyper-dissipative system. The global existence of classical solutions with $d \geq 3$ can only been obtained when $\alpha>\frac{d+2}{4}[22,37]$. However, the existence of classical global solution to the two-dimensional Navier-Stokes equations can be solved readily due to the validity of the energy inequality in the $L^{2}$ space [20]. Actually, similar to the ideal flow governed by the two-dimensional Euler equations, the maximum principle holds true for the vorticity formulation of the two-dimensional generalized Navier-Stokes equations. This gives rise to the additional $L_{\infty}$ estimate of the corresponding vorticity field and hence the existence of the global classical solutions to the two-dimensional equations for $\alpha \geq 0$ (see, for example, [6]). This global solution existence can be extended from that of the two-dimensional Euler equations $[5,19,41]$. For the well-posedness with respect to the existence and uniqueness of small global solutions or small time solutions to (1) with $\alpha>\frac{1}{2}$, we refer to [39] for solutions in critical homogeneous Besov spaces and [28] for solutions in critical homogeneous $Q$ spaces introduced in $[28,40]$. For the limit case of $\alpha=0$ and $d=2$, equation (1) represents actually the simplified atmospheric circulation equations system of Charney and DeVore 
$[7,9,12,35]$. The vorticity formulation of (1) with $d=2$ is similar to the surface dissipative quasi-geostrophic equation $[6,10,24,36]$. However, the vorticity-velocity relations $\omega=\nabla \times u$ in the former and $\theta=\nabla^{\perp}(-\Delta)^{-1 / 2} u$ in the latter lead to the difference for the global solution existence of the two systems. For the problem on the nonexistence of the global solutions to the supercritical surface dissipative quasi-geostrophic equation, one may refer to [23].

A homogeneous Besov space is a collection of the functions $f$ which can be expressed as the Littlewood-Paley decomposition

$$
f=\sum_{-\infty<j<\infty} \Delta_{j} f
$$

for the dyadic blocks $\Delta_{j}$. This unit decomposition helps define the norm of the homogeneous Besov space and examine the function $f$ by reducing to the examination of individual terms $\Delta_{j} f$ through Bernstein inequalities (see, for example, [26]). The Littlewood-Paley decomposition approach is overwhelming in the analytic study of fluid mechanics equations (see, for example, [14] for the compressible Navier-Stokes equations, [15] for the density-dependent incompressible Navier-Stokes equations, [38, 39] for the generalized Navier-Stokes euqations (1), and [8] for the dissipative quasi-geostrophic equation).

Note that the fractional Laplacian $(-\Delta)^{\alpha}$ generates an analytic semigroup operator $\mathrm{e}^{-t(-\Delta)^{\alpha}}$, which can be characterized in homogeneous Besov spaces in terms of the LittlewoodPaley decomposition [33, 34]. This characterization simplifies the derivation of a priori estimates of the system (1) and was applied to the understanding of the well-posedness of the Navier-Stokes equations [11]. The purpose of the present paper is to extend this approach to the generalized Navier-Stokes equations (1) and then to provide a new approach to the understanding of a higher regularity problem initiated from [18].

For simplicity, the notation $\lesssim$ is adopted in the sense that $a_{1} \lesssim a_{2}$ represents the inequality $a_{1} \leq C a_{2}$ for a constant $C$ independent of the quantities $t \in[0, T), x \in R^{d}$, $T>0$, functions $u, v, f, g$ and a scalar constant $\lambda>0$. Moreover, we use the equivalence relation $a_{1} \simeq a_{2}$ if both $a_{1} \lesssim a_{2}$ and $a_{2} \lesssim a_{1}$ hold ture.

As is well known, equation (1) is scale invariant with respect to the scale transformation

$$
u_{\lambda}(t, x)=\lambda^{2 \alpha-1} u\left(\lambda^{2 \alpha} t, \lambda x\right), \quad \pi_{\lambda}(t, x)=\lambda^{4 \alpha-2} \pi\left(\lambda^{2 \alpha} t, \lambda x\right) .
$$

That is, equation (1) is equivalent to the scaled equations

$$
\partial_{t} u_{\lambda}+(-\Delta)^{\alpha} u_{\lambda}+u_{\lambda} \cdot \nabla u_{\lambda}+\nabla \pi_{\lambda}=0, \nabla \cdot u_{\lambda}=0 .
$$

According to this scale-invariant property, it is beneficial to examine equation (1) in homogeneous function spaces. A spatial function space $X^{s}\left(R^{d}\right)$ is said to be homogeneous of degree $s$ if

$$
\|f(\lambda \cdot)\|_{X^{s}\left(R^{d}\right)} \simeq \lambda^{s}\|f\|_{X^{s}\left(R^{d}\right)} \text { for } f \in X^{s}
$$

and a temporal-spacial function space $Y^{s}\left([0, T) \times R^{d}\right)$ for $0<T \leq \infty$ is said to be homogeneous of degree $s$ if

$$
\left\|f\left(\lambda^{2 \alpha} \cdot \lambda \cdot\right)\right\|_{Y^{s}\left([0, T) \times R^{d}\right)} \simeq \lambda^{s}\|f\|_{Y^{s}\left(\left[0, \lambda^{2 \alpha} T\right) \times R^{d}\right)} \text { for } f \in Y^{s}\left([0, T) \times R^{d}\right)
$$

for $-\infty<s<\infty$ and $\lambda>0$.

For the integral formulation of (1) transformed as

$$
u+\left(\partial_{t}+(-\Delta)^{\alpha}\right)^{-1}(u \cdot \nabla u+\nabla \pi)=0, \quad \nabla \cdot u=0
$$

initially from the static state $u(0)=0$, an analytic semigroup approach is applied to equation (3) on a homogeneous space $Y^{s}$ involving a homogeneous Besov space to obtain the estimate

$$
\|u\|_{Y^{s}} \lesssim\left\|\left(\partial_{t}+(-\Delta)^{\alpha}\right)^{-1}(u \cdot \nabla u)\right\|_{Y^{s}} \lesssim\|u u\|_{Y^{s+1-2 \alpha}} \lesssim\|u\|_{Y^{s}}\|u\|_{Y^{1-2 \alpha}} .
$$

This together with a linearization technique implies that the homogeneous space $Y^{s}$ with $s=1-2 \alpha$ is the critical space for ensuring the unique existence of the solution to (1). Moreover, it is obtained that the estimates involve an unbounded exponential differential 
operator or an inverse of an analytic semigroup operator. This is known as the Gevrey regularity problem examined in [18] for Navier-Stokes equations in Hilbert spaces and is further developed in $[4,26]$. For the recent investigations of this problem in dissipative quasi-geostrophic equations and some other related dissipative equations, one may refer to $[1,2,16,17]$.

The significance of the present paper is the development of an analytic semigroup approach in Besov spaces for the well-posedness and Gevrey regularity problem of the generalized Navier-Stokes equations (1) and provides an alternative way for the understanding of the problem different to the Bernstein estimates approach based on the dyadic Littlewood-Paley blocks in earlier studies (see, for example, [39]).

This paper is organized as follows. Firstly, we display basic properties of Besov spaces and its connection with the analytic semigroup $\mathrm{e}^{-t(-\Delta)^{\alpha}}$ in Section 2. Next, we derive a priori estimates of linearized equation of (1) in homogeneous Besov spaces by using the semigroup approach. Finally, the result obtained for the linearized system is extended to the nonlinear equation (1) to show the well-posedness and Gevrey regularity properties.

\section{BESOV SPACES AND BASIC PROPERTIES}

For the readers' convenience, we collect basic properties of Besov spaces and their homogeneous counterparts from $[33,34]$.

Standard notion is used. $S\left(R^{d}\right)$ represents the Schwartz space of rapidly decreasing infinitely differential functions on $R^{d}$. Its dual space $S^{\prime}\left(R^{d}\right)$ is the collection of all tempered distributions on $R^{d}$. $L_{p}\left(R^{d}\right)$ denotes the Lebesgue space of functions on $R^{d}$ for $1 \leq p \leq \infty$. $F$ represents the Fourier transform in $S^{\prime}\left(R^{d}\right)$ satisfying $F f(\xi)=\int_{R^{d}} \mathrm{e}^{-\mathrm{i} x \cdot \xi} f(x) d x$ and the inverse Fourier transform is defined as $F^{-1}$.

As in [33, Definitions 2.3.1/1, 2.3.1/2 and 5.1.3/2], let $\phi$ be a smooth function on $R^{d}$ in the following sense $\phi(\xi)=0$ for $|\xi| \geq 2$ and $\phi(\xi)=1$ for $|\xi| \leq 1$ which produces the dyadic block symbols

and the dyadic blocks

$$
\psi_{j}(\xi)=\phi\left(2^{-j} \xi\right)-\phi\left(2^{-j+1} \xi\right)
$$

$$
\Delta_{j}=F^{-1} \psi_{j} F
$$

for $j \in Z$, the integer set. Therefore, for $1 \leq p, q \leq \infty$ and $-\infty<s<\infty$, we define the Besov space

$$
B_{p, q}^{s}\left(R^{d}\right)=\left\{f \in S^{\prime}\left(R^{d}\right) ;\|f\|_{B_{p, q}^{s}}=\left(\left\|F^{-1} \phi F f\right\|_{L_{p}}^{q}+\sum_{j=1}^{\infty} 2^{j s q}\left\|\Delta_{j} f\right\|_{L_{p}}^{q}\right)^{\frac{1}{q}}<\infty\right\},
$$

and the homogeneous Besov space

$\dot{B}_{p, q}^{s}\left(R^{d}\right)=\left\{f \in S^{\prime}\left(R^{d}\right) ; f=\sum_{j=-\infty}^{\infty} \Delta_{j} f,\|f\|_{\dot{B}_{p, q}^{s}}=\left(\sum_{j=-\infty}^{\infty} 2^{j s q}\left\|\Delta_{j} f\right\|_{L_{p}}^{q}\right)^{\frac{1}{q}}<\infty\right\}$.

Here the $l_{q}$ norm reduces to the $l_{\infty}$ norm for the sequences $\left\{2^{j s}\left\|\Delta_{j} f\right\|_{L_{p}}\right\}_{j \in N}$ and $\left\{2^{j s}\left\|\Delta_{j} f\right\|_{L_{p}}\right\}_{j \in Z}$ when $q=\infty$.

It is readily seen that the space $\dot{B}_{p, q}^{s}\left(R^{d}\right)$ is homogeneous of degree $s-\frac{d}{p}$ and its norm can be expressed as

$$
\|f\|_{\dot{B}_{p, q}^{s}}=\lim _{k \rightarrow \infty} \frac{\|f(\lambda \cdot)\|_{B_{p, q}^{s}}}{\lambda^{s-\frac{d}{p}}}, \quad \lambda=2^{k}
$$

for $s>0$ and $f \in B_{p, q}^{s}\left(R^{d}\right)$.

Lemma 1. ([33, Theorem 5.2.3/1]) Let $-\infty<\sigma, s<\infty$ and $1 \leq p, q \leq \infty$. Then the fractional operator $(-\Delta)^{\frac{\sigma}{2}}$ maps $\dot{B}_{p, q}^{s}\left(R^{d}\right)$ isomorphically onto $\dot{B}_{p, q}^{s+\sigma}\left(R^{d}\right)$ or there holds the 
norm equivalence

$$
\left\|(-\Delta)^{\frac{\sigma}{2}} f\right\|_{\dot{B}_{p, q}^{s}} \simeq\|f\|_{\dot{B}_{p, q}^{s+\sigma}} \text { for } f \in \dot{B}_{p, q}^{s+\sigma}\left(R^{d}\right) .
$$

The real interpolation property of Besov spaces [33] gives the following interpolation inequality.

Lemma 2. ([33, Theorem 2.4.2 and Subsection 5.2.5]) Let $0<\theta<1,1 \leq p, q_{0}, q_{1}, q \leq \infty$, $-\infty<s<\tau<\infty$ and $\sigma=(1-\theta) s+\theta \tau$. Then there holds the real interpolation property

$$
\dot{B}_{p, q}^{\sigma}\left(R^{d}\right)=\left(\dot{B}_{p, q_{0}}^{s}\left(R^{d}\right), \dot{B}_{p, q_{1}}^{\tau}\left(R^{d}\right)\right)_{\theta, q}
$$

and

$$
\|f\|_{\dot{B}_{p, q}^{\sigma}} \lesssim\|f\|_{\dot{B}_{p, q_{0}}^{s}}^{1-\theta}\|f\|_{\dot{B}_{p, q_{1}}^{\tau}}^{\theta}
$$

for $f \in \dot{B}_{p, q_{0}}^{s}\left(R^{d}\right) \cap \dot{B}_{p, q_{1}}^{\tau}\left(R^{d}\right)$.

The combination of equation (5) and the characterization of the Besov space $B_{p, \infty}^{s}\left(R^{d}\right)$ (see [33, Eqs 2.3.5/3, Theorem 2.5.12]) by the difference operators

$$
\triangle_{h}^{0} f(x)=f(x), \triangle_{h}^{1} f(x)=f(x+h)-f(x), \triangle_{h}^{k} f(x)=\triangle_{h}^{1}\left(\triangle_{h}^{k-1} f(x)\right) \text { for } h \in R^{d}
$$

produces the following characterization of its homogeneous counterpart.

Lemma 3. Let $s>0,1 \leq p \leq \infty$ and an integer $k>s$. Then there holds the equivalence relation

$$
\|f\|_{\dot{B}_{p, \infty}^{s}} \simeq \operatorname{essipp}_{0 \neq h \in R^{d}} \frac{\left\|\triangle_{h}^{k} f\right\|_{L_{p}}}{|h|^{s}} \text { for } f \in \dot{B}_{p, \infty}^{s}\left(R^{d}\right)
$$

The operator $\mathrm{e}^{-t(-\Delta)^{\alpha}}=F^{-1} \mathrm{e}^{-t|\xi|^{2 \alpha}} F$ is an analytic semigroup in $L_{p}\left(R^{d}\right)$ generated by the fractional Laplacian $(-\Delta)^{\alpha}=F^{-1}|\xi|^{2 \alpha} F$, which maps the fractional Sobolev space $H_{p}^{2 \alpha}\left(R^{d}\right)=\left(1+(-\Delta)^{\alpha}\right)^{-1} L_{p}\left(R^{d}\right)$ onto $L_{p}\left(R^{d}\right)$, due to the validity of the property

$$
\left\|(-\Delta)^{\alpha} \mathrm{e}^{-t(-\Delta)^{\alpha}} f\right\|_{L_{p}} \lesssim t^{-1}\|f\|_{L_{p}} \text { for } t>0 .
$$

Therefore, for $1<p<\infty$ and $k \geq 1$, the characterization property [34, Proposition 1.8.1/1]

$$
\left(L_{p}\left(R^{d}\right), H_{p}^{2 \alpha k}\left(R^{d}\right)\right)_{\theta, \infty}=\left\{f \in L_{p}\left(R^{d}\right) ; \sup _{t>0} t^{k-k \theta}\left\|(-\Delta)^{\alpha k} \mathrm{e}^{-t(-\Delta)^{\alpha}} f\right\|_{L_{p}}<\infty\right\}
$$

and the real interpolation property [33, Theorems 2.4.2 and 2.5.6]

$$
\left(L_{p}\left(R^{d}\right), H_{p}^{2 \alpha k}\left(R^{d}\right)\right)_{\theta, \infty}=B_{p, \infty}^{2 \alpha k \theta}\left(R^{d}\right)
$$

yield the characterization of the Besov space $B_{p, \infty}^{s}\left(R^{d}\right)$

$$
\|f\|_{B_{p, \infty}^{s}} \simeq\|f\|_{L_{p}}+\sup _{t>0} t^{k-\frac{s}{2 \alpha}}\left\|(-\Delta)^{\alpha k} \mathrm{e}^{-t(-\Delta)^{\alpha}} f\right\|_{L_{p}}
$$

for $s>0,1<p<\infty$, and $k>\frac{s}{2 \alpha}$. Using (5) to remove the lower order part $\|f\|_{L_{p}}$ of (10), we obtain the characterization of the homogeneous Besov space by the analytic semigroup $\mathrm{e}^{-t(-\Delta)^{\alpha}}$ in the following.

Lemma 4. Let $\alpha>0, s>0,1<p<\infty$ and an integer $k>s /(2 \alpha)$. Then we have

$$
\|f\|_{\dot{B}_{p, \infty}^{s}} \simeq \sup _{t>0} t^{k-\frac{s}{2 \alpha}}\left\|(-\Delta)^{\alpha k} \mathrm{e}^{-t(-\Delta)^{\alpha}} f\right\|_{L_{p}} \text { for } f \in \dot{B}_{p, \infty}^{s}\left(R^{d}\right) .
$$

Thus the homogeneous Besov space defined by the Littlewood-Paley decomposition reduces to that characterized by the analytic semigroup $\mathrm{e}^{-t(-\Delta)^{\alpha}}$ generated by $(-\Delta)^{\alpha}$.

Lemma 5. ([26, Lemma 24.8]) Let $d \geq 1,1<p, q<\infty, \frac{1}{r}=\frac{1}{p}+\frac{1}{q}, f \in L_{p}\left(R^{d}\right)$ and $g \in L_{q}\left(R^{d}\right)$. Then there holds the inequality

$$
\left\|\mathrm{e}^{t|\nabla|_{1}}\left(\left(\mathrm{e}^{-t|\nabla|_{1}} f\right) \mathrm{e}^{-t|\nabla|_{1}} g\right)\right\|_{L_{r}} \lesssim\|f\|_{L_{p}}\|g\|_{L_{q}},
$$

where the operator $\mathrm{e}^{\frac{1}{2 \alpha}}|\nabla|_{1}=F^{-1} \mathrm{e}^{\frac{1}{2 \alpha}}|\xi|_{1} F$ by using the $l_{1}$ norm $|\xi|_{1}=\sum_{i=1}^{d}\left|\xi_{i}\right|$. 
This generalized Hölder inequality given in [26] is essential in the investigation of Gevrey regularity problem. For the readers' convenience, it is demonstrated as follows.

Proof. Let begin with the case $d=1$. For $|\nabla|=|\nabla|_{1}, \hat{f}=F f$ and real variables $x, y, \eta, \xi \in$ $R$ in the one-dimensional situation, we have

$$
\begin{aligned}
\mathrm{e}^{t|\nabla|}\left(\mathrm{e}^{-t|\nabla|} f \mathrm{e}^{-t|\nabla|} g\right)(x) & \int_{R^{2}} \mathrm{e}^{\mathrm{i} x(\xi+\eta)} \mathrm{e}^{t|\xi+\eta|-t|\eta|-t|\xi|} \hat{f}(\eta) \hat{g}(\xi) d \eta d \xi \\
= & \int_{\eta \xi \geq 0} \mathrm{e}^{\mathrm{i} x \cdot(\xi+\eta)} \hat{f}(\eta) \hat{g}(\xi) d \eta d \xi+\int_{\eta \xi<0} \mathrm{e}^{\mathrm{i} x(\xi+\eta)} \mathrm{e}^{t|\xi+\eta|-t|\eta|-t|\xi|} \hat{f}(\eta) \hat{g}(\xi) d \eta d \xi \\
= & \int_{\eta \xi \geq 0} \mathrm{e}^{\mathrm{i} x(\xi+\eta)}\left(1-\mathrm{e}^{-2 t|\xi|}\right) \hat{f}(\eta) \hat{g}(\xi) d \eta d \xi \\
& +\left(\int_{0}^{\infty} d \eta \int_{-\infty}^{-\eta} d \xi+\int_{-\infty}^{0} d \eta \int_{-\eta}^{\infty} d \xi\right) \mathrm{e}^{\mathrm{i} x(\xi+\eta)} \mathrm{e}^{-2 t|\eta|} \hat{f}(\eta) \hat{g}(\xi) \\
& +\left(\int_{0}^{\infty} d \eta \int_{-\eta}^{\infty} d \xi+\int_{-\infty}^{0} d \eta \int_{-\infty}^{-\eta} d \xi\right) \mathrm{e}^{\mathrm{i} x(\xi+\eta)} \mathrm{e}^{-2 t|\xi|} \hat{f}(\eta) \hat{g}(\xi) \\
= & \int_{\eta \xi \geq 0} \mathrm{e}^{\mathrm{i} x(\xi+\eta)}\left(1-\mathrm{e}^{-2 t|\xi|}\right) \hat{f}(\eta) \hat{g}(\xi) d \eta d \xi+\int_{\xi \eta<0} \mathrm{e}^{\mathrm{i} x \xi} \mathrm{e}^{-2 t|\eta|} \hat{f}(\eta) \hat{g}(\xi-\eta) d \eta d \xi \\
& +\int_{\xi \eta>0} \mathrm{e}^{\mathrm{i} x \xi} \mathrm{e}^{-2 t|\xi-\eta|} \hat{f}(\eta) \hat{g}(\xi-\eta) d \eta d \xi \doteq I_{1}+I_{2}+I_{3} .
\end{aligned}
$$

Defining the half space Fourier transforms

$$
S_{+} f=\int_{0}^{\infty} \mathrm{e}^{\mathrm{i} x \xi} \hat{f} d x \quad \text { and } \quad S_{-} f=\int_{-\infty}^{0} \mathrm{e}^{\mathrm{i} x \xi} \hat{f} d x
$$

we have

$$
\begin{gathered}
I_{1}=\int_{0}^{\infty} \mathrm{e}^{\mathrm{i} x \eta} \hat{f}(\eta) d \eta \int_{0}^{\infty} \mathrm{e}^{\mathrm{i} x \xi}\left(1-\mathrm{e}^{-2 t|\xi|}\right) \hat{g}(\xi) d \xi+\int_{-\infty}^{0} \mathrm{e}^{\mathrm{i} x \eta} \hat{f}(\eta) d \eta \int_{-\infty}^{0} \mathrm{e}^{\mathrm{i} x \xi}\left(1-\mathrm{e}^{-2 t|\xi|}\right) \hat{g}(\xi) d \xi \\
=\left(S_{+} f\right) S_{+}\left(1-\mathrm{e}^{-2 t|\nabla|}\right) g+\left(S_{-} f\right) S_{-}\left(1-\mathrm{e}^{-2 t|\nabla|}\right) g \\
I_{2}=\int_{-\infty}^{0} \mathrm{e}^{\mathrm{i} x \xi} F\left(g(y) \int_{0}^{\infty} \mathrm{e}^{\mathrm{i} y \eta} \mathrm{e}^{-2 t|\eta|} \hat{f}(\eta) d \eta\right)(\xi) d \xi \\
\\
\quad+\int_{0}^{\infty} \mathrm{e}^{\mathrm{i} x \xi} F\left(g(y) \int_{-\infty}^{0} \mathrm{e}^{\mathrm{i} y \eta} \mathrm{e}^{-2 t|\eta|} \hat{f}(\eta) d \eta\right)(\xi) d \xi \\
=S_{-}\left(g S_{+} \mathrm{e}^{-2 t|\nabla|} f\right)+S_{+}\left(g S_{-} \mathrm{e}^{-2 t|\nabla|} f\right)
\end{gathered}
$$

and, similarly,

$$
I_{3}=S_{+}\left(f S_{+} \mathrm{e}^{-2 t|\nabla|} g\right)+S_{-}\left(f S_{-} \mathrm{e}^{-2 t|\nabla|} g\right)
$$

Note that

$$
\left\|S_{ \pm} f\right\| \lesssim\|f\|_{L_{\tau}} \text { and }\left\|\mathrm{e}^{-2 t|\nabla|_{1}} f\right\|_{L_{\tau}} \lesssim\|f\|_{L_{\tau}}
$$

for $\tau=r, p, q$ since the operators $S_{ \pm}$are $L_{\tau}$ Fourier multiplier operators [30, Theorem 4.1.4] and $\mathrm{e}^{-2 t|\nabla|_{1}}$ is obviously an $L_{\tau}$ Fourier multiplier operator. We thus use Hölder inequality and estimates (13) to obtain the validity of (12) in the case $d=1$. When $d>1$, the observation $\mathrm{e}^{t|\nabla|_{1}}=\mathrm{e}^{t\left|\partial_{x_{1}}\right|} \mathrm{e}^{t\left|\partial_{x_{2}}\right|} \ldots \mathrm{e}^{t\left|\partial_{x_{d}}\right|}$ ensures that the $d$-dimensional operator is decomposed into 1-dimensional operators. Thus there exist $L_{\tau}$ Fourier multiplier operators $L_{i}, M_{i}$ and $N_{i}$ given by $S_{ \pm}$and $\mathrm{e}^{-2 t|\nabla|_{1}}$ so that

$$
\mathrm{e}^{t|\nabla|_{1}}\left(\left(\mathrm{e}^{-t|\nabla|_{1}} f\right) \mathrm{e}^{-t|\nabla|_{1}} g\right)=\sum_{i=1}^{3 d} L_{i}\left(\left(N_{i} g\right) M_{i} f\right) .
$$


This together with the estimates (13) and the Hölder inequality implies

$$
\left\|\mathrm{e}^{t|\nabla|_{1}}\left(\left(\mathrm{e}^{-t|\nabla|_{1}} f\right) \mathrm{e}^{-t|\nabla|_{1}} g\right)\right\|_{L_{r}} \lesssim \sum_{i=1}^{3 d}\left\|\left(N_{i} g\right) M_{i} f\right\|_{L_{r}} \lesssim\|g\|_{L_{p}}\|f\|_{L_{q}}
$$

and completes the proof of (12) for $d>1$.

\section{Analytic Semigroup approach to Generalized Stokes equations}

In this paper, the existence of local solutions and small solutions of the nonlinear system (1) is examined and can be obtained by a linearization technique. Therefore, it is beneficial to consider the linearized system of (1) or the generalized Stokes equations

$$
\partial_{t} u+\nabla \pi+(-\Delta)^{\alpha} u=\nabla \cdot f, \nabla \cdot u=0,
$$

coupled with zero initial condition

$$
u(0)=0 \text { in } R^{d} .
$$

Here $f: R^{d} \mapsto R^{d^{2}},(\nabla \cdot f)_{j}=\sum_{i=1}^{d} \partial_{i} f_{i j}$ and the pressure force term $\nabla \pi$ can be written as

$$
\nabla \pi=\nabla \Delta^{-1} \nabla \cdot(\nabla \cdot f)
$$

due to divergence property of $u$. Hence (14) is written as

$$
\partial_{t} u+(-\Delta)^{\alpha} u=P \nabla \cdot f \text { in }(0, T) \times R^{d},
$$

for the bounded projection operator

$$
P=\delta_{i j}-\partial_{i} \partial_{j} \Delta^{-1}
$$

in $L_{p}\left(R^{d}\right)$ for $1<p<\infty$, where $\delta_{i j}$ denotes the Kronecker symbol. Now we rewrite the differential equation (16) in the form of the integral equation

$$
u(t)=\left(\partial_{t}+(-\Delta)^{\alpha}\right)^{-1} P \nabla \cdot f=\int_{0}^{t} \mathrm{e}^{-(t-s)(-\Delta)^{\alpha}} P \nabla \cdot f(s) d s .
$$

With the use of the characterization by the analytic semigroup, the purpose of this section is to show that the operators $\left(\partial_{t}+(-\Delta)^{\alpha}\right)^{-1} P \nabla$ behaviours like the operator $\left.|\nabla|\right|^{1-2 \alpha}$ mapping a homogeneous space of degree $s$ into a homogeneous space of degree $s+1-2 \alpha$.

Theorem 1. Let $d \geq 2,0<T \leq \infty, 0 \leq \beta<\alpha,-2 \beta<\sigma$ and $1<p<\infty$. Define the operators $S(t)=F^{-1} m(t, \xi) F$ and $S^{-1}(t)=F^{-1} \frac{1}{m(t, \xi)} F$ for a symbol $m(t, \cdot)$. Assume that the inequality

$$
\left\|\mathrm{e}^{-\frac{1}{4}(t-s)(-\Delta)^{\alpha}} S(t) S^{-1}(s) g\right\|_{L_{p}} \lesssim\|g\|_{L_{p}}
$$

holds true for $g \in L_{p}\left(R^{d}\right)$. Then $u(t)$ presented in equation (17) is subject to the estimates

$$
\|S(t) u(t)\|_{\dot{B}_{p, \infty}^{\sigma}}+t^{\frac{\beta}{\alpha}}\|S(t) u(t)\|_{\dot{B}_{p, \infty}^{\sigma+2 \beta}} \lesssim \operatorname{esssup}_{0<t<T} t^{\frac{\beta}{\alpha}}\|S(t) f(t)\|_{\dot{B}_{p, \infty}^{\sigma+2 \beta+1-2 \alpha}}
$$

and

$$
\|S(t) u(t)\|_{L_{\infty}} \lesssim \operatorname{esssup}_{0<t<T} t^{\frac{\beta}{\alpha}}\|S(t) f(t)\|_{\dot{B}_{p, \infty}^{2 \beta+2 \alpha+\frac{d}{p}}}
$$

for $0<t<T$, provided that the right-hand sides are finite.

A typical example of the operator $S(t)$ is $\mathrm{e}^{t^{\frac{\gamma}{\alpha}}(-\Delta)^{\gamma}}$ for $0 \leq \gamma<\alpha$. 
Proof. For an integer $k>\frac{\sigma+2 \beta}{2 \alpha}$, it follows from Lemmas 1 and 4 , equations (9) and (18) and the boundedness of the projection operator $P$ in the space $L_{p}\left(R^{d}\right)$ that

$$
\begin{aligned}
\|S(t) u(t)\|_{\dot{B}_{p, \infty}^{\sigma+2 \beta}} \\
\simeq \sup _{\tau>0} \tau^{k-\frac{\sigma+2 \beta}{2 \alpha}}\left\|(-\Delta)^{\alpha k} \mathrm{e}^{-\tau(-\Delta)^{\alpha}} \int_{0}^{t} P S(t) S^{-1}(s) \mathrm{e}^{-(t-s)(-\Delta)^{\alpha}} S(s) \nabla \cdot f(s) d s\right\|_{L_{p}} \\
\lesssim \sup _{\tau>0} \tau^{k-\frac{\sigma+2 \beta}{2 \alpha}} \int_{0}^{t}\left\|(-\Delta)^{\alpha k} \mathrm{e}^{-\frac{3}{4}(t-s+\tau)(-\Delta)^{\alpha}} S(s) \nabla \cdot f(s)\right\|_{L_{p}} d s \\
\lesssim \sup _{\tau>0} \tau^{k-\frac{\sigma+2 \beta}{2 \alpha}} \int_{0}^{t}(t-s+\tau)^{-1}\left\|(-\Delta)^{\alpha k} \mathrm{e}^{-\frac{1}{2}(t-s+\tau)(-\Delta)^{\alpha}}(-\Delta)^{-\alpha} S(s) \nabla \cdot f(s)\right\|_{L_{p}} d s \\
\lesssim \sup _{\tau>0} \tau^{k-\frac{\sigma+2 \beta}{2 \alpha}} \int_{0}^{t}(t-s+\tau)^{-k-1+\frac{\sigma+2 \beta}{2 \alpha}} s^{-\frac{\beta}{\alpha}} s^{\frac{\beta}{\alpha}}\left\|(-\Delta)^{-\alpha} S(s) \nabla \cdot f(s)\right\|_{\dot{B}_{p, \infty}^{\sigma+2 \beta}} d s .
\end{aligned}
$$

After the calculation of the integral over the intervals $\left[0, \frac{t}{2}\right)$ and $\left[\frac{t}{2}, t\right)$, the previous inequality reduces to

$$
\begin{aligned}
\|S(t) u\|_{\dot{B}_{p, \infty}^{\sigma+2 \beta}} \lesssim & \sup _{\tau>0} \tau^{k-\frac{\sigma+2 \beta}{2 \alpha}}\left[(t+\tau)^{-k+\frac{\sigma+2 \beta}{2 \alpha}} t^{-\frac{\beta}{\alpha}}+(t+\tau)^{-k-1+\frac{\sigma+2 \beta}{2 \alpha}} t^{1-\frac{\beta}{\alpha}}\right] \\
& \quad \cdot \quad \underset{0<t<T}{\operatorname{ess} \sup } t^{\frac{\beta}{\alpha}}\|S(t) f(t)\|_{\dot{B}_{p, \infty}^{\sigma+2 \beta+1-2 \alpha}} \\
\lesssim & t^{-\frac{\beta}{\alpha}} \underset{0<t<T}{\operatorname{ess} \sup } t^{\frac{\beta}{\alpha}}\|S(t) f(t)\|_{\dot{B}_{p, \infty}^{\sigma+2 \beta+1-2 \alpha}} .
\end{aligned}
$$

Furthermore, equation (9) and the interpolation inequality

$$
\left\|(-\Delta)^{\gamma} \mathrm{e}^{-t(-\Delta)^{\alpha}} g\right\|_{L_{p}} \lesssim\left\|\mathrm{e}^{-t(-\Delta)^{\alpha}} g\right\|_{L_{p}}^{1-\frac{\gamma}{\alpha}}\left\|(-\Delta)^{\alpha} \mathrm{e}^{-t(-\Delta)^{\alpha}} g\right\|_{L_{p}}^{\frac{\gamma}{\alpha}}
$$

imply that

$$
\left\|(-\Delta)^{\gamma} \mathrm{e}^{-t(-\Delta)^{\alpha}} g\right\|_{L_{p}} \lesssim t^{-\frac{\gamma}{\alpha}}\|g\|_{L_{p}},
$$

and hence

$$
\left\|(-\Delta)^{\gamma} \mathrm{e}^{-t(-\Delta)^{\alpha}} g\right\|_{\dot{B}_{p, \infty}^{s}} \lesssim t^{-\frac{\gamma}{\alpha}}\|g\|_{\dot{B}_{p, \infty}^{s}} .
$$

Using Lemma 1, equations (18) and (23) and the boundedness of the operator $P$, we have

$$
\begin{aligned}
& \|S(t) u(t)\|_{\dot{B}_{p, \infty}^{\sigma}} \lesssim \quad \int_{0}^{t}\left\|\mathrm{e}^{-\frac{3}{4}(t-s)(-\Delta)^{\alpha}} S(s) \nabla \cdot f(s)\right\|_{\dot{B}_{p, \infty}^{\sigma}} d s \\
& \lesssim \int_{0}^{t}(t-s)^{-1+\frac{\beta}{\alpha}}\left\|(-\Delta)^{-(\alpha-\beta)} S(s) \nabla \cdot f(s)\right\|_{\dot{B}_{p, \infty}^{\sigma}} d s \\
& \lesssim \quad \int_{0}^{t}(t-s)^{-1+\frac{\beta}{\alpha}} s^{-\frac{\beta}{\alpha}} d s \underset{0<t<T}{\operatorname{ess} \sup } t^{\frac{\beta}{\alpha}}\|S(t) f(t)\|_{\dot{B}_{p, \infty}^{\sigma+2 \beta+1-2 \alpha}} \\
& \lesssim \quad \operatorname{esssup}_{0<t<T} t^{\frac{\beta}{\alpha}}\|S(t) f(t)\|_{\dot{B}_{p, \infty}^{\sigma+2 \beta+1-2 \alpha}} .
\end{aligned}
$$

To derive the estimate in the $L_{\infty}$ norm, we employ the Gagaliardo-Nirenberg inequality [29] to obtain that

$$
\left\|\mathrm{e}^{-t(-\Delta)^{\alpha}} g\right\|_{L_{\infty}} \lesssim\left\|\mathrm{e}^{-t(-\Delta)^{\alpha}} g\right\|_{L_{q}}^{1-\frac{d}{q}}\left\|\nabla \mathrm{e}^{-t(-\Delta)^{\alpha}} g\right\|_{L_{q}}^{\frac{d}{q}} \lesssim t^{-\frac{d}{2 \alpha q}}\|g\|_{L_{q}} .
$$


for a real number $q>d$. If the number $q>p$ satisfies additionally the condition $2 \beta+\frac{d}{q}<2 \alpha$, equations (6), (11), (18), (24) and (22) yield

$$
\begin{aligned}
\|S(t) u(t)\|_{L_{\infty}} & \lesssim \int_{0}^{t}(t-s)^{-\frac{d}{2 \alpha q}}\left\|\mathrm{e}^{-\frac{3}{4}(t-s)(-\Delta)^{\alpha}} S(t) P \nabla \cdot f(s)\right\|_{L_{q}} d s \\
& \lesssim \int_{0}^{t}(t-s)^{-\frac{d}{2 \alpha q}}\left\|\mathrm{e}^{-\frac{1}{2}(t-s)(-\Delta)^{\alpha}} S(s) \nabla \cdot f(s)\right\|_{L_{q}} d s \\
& \lesssim \int_{0}^{t}(t-s)^{-\frac{d}{2 \alpha q}}(t-s)^{-1+\frac{2 \beta+\frac{d}{q}}{2 \alpha}}\left\|S(s)(-\Delta)^{-\alpha} \nabla \cdot f(s)\right\|_{\dot{B}_{q, \infty}^{2 \beta+\frac{d}{q}}} d s \\
& \lesssim \int_{0}^{t}(t-s)^{-1+\frac{\beta}{\alpha}} s^{-\frac{\beta}{\alpha}} d s \underset{0<t<T}{\operatorname{ess} \sup } t^{\frac{\beta}{\alpha}}\|S(t) f(t)\|_{\dot{B}_{q, \infty}^{2 \beta+1-2 \alpha+\frac{d}{q}}} \\
& \lesssim \quad \underset{0<t<T}{\operatorname{ess} \sup } t^{\frac{\beta}{\alpha}}\|S(t) f(t)\|_{\dot{B}_{p, \infty}^{2 \beta+2 \alpha+\frac{d}{p}}},
\end{aligned}
$$

where we have used the imbedding theorem [33, Theorem 2.7.1 and Subsection 5.2.5]

$$
\dot{B}_{p, \infty}^{\gamma+\frac{d}{p}}\left(R^{d}\right) \hookrightarrow \dot{B}_{q, \infty}^{\gamma+\frac{d}{q}}\left(R^{d}\right) .
$$

Collecting terms, we have the desired estimate and thus complete the proof of Theorem 1 .

The symbol $m=\mathrm{e}^{-\frac{1}{4}(t-s)|\xi|^{2 \alpha}+t^{\frac{1}{2 \alpha}}|\xi|_{1}-s^{\frac{1}{2 \alpha}}|\xi|_{1}}$ satisfies the conditions of the Marcinkiewicz multiplier theorem [30, Theorem 6.1.6'] and thus there holds the $L_{p}$ estimate

$$
\left\|\mathrm{e}^{-\frac{1}{4}(t-s)(-\Delta)^{\alpha}} \mathrm{e}^{\frac{1}{2 \alpha}|\nabla|_{1}} \mathrm{e}^{-s^{\frac{1}{2 \alpha}}|\nabla|_{1}} g\right\|_{L_{p}} \lesssim\|g\|_{L_{p}} \text { for } \alpha>\frac{1}{2},
$$

which gives an example of $S(t)=\mathrm{e}^{\frac{1}{2 \alpha}|\nabla|_{1}}$ in (18). For this special choice, Theorem 1 can be stated as

Corollary 1. Let $d \geq 2, \frac{1}{2}<\alpha, 0 \leq \beta<\alpha, 1<p<\infty$ and $0<\sigma+2 \beta$. Then $u=\left(\partial_{t}-(-\Delta)^{\alpha}\right)^{-1} P \nabla \cdot f$ is subject to the estimate

$$
\left\|\mathrm{e}^{\frac{1}{2 \alpha}|\nabla|_{1}} u(t)\right\|_{\dot{B}_{p, \infty}^{\sigma}}+t^{\frac{\beta}{\alpha}}\left\|\mathrm{e}^{\frac{1}{2 \alpha}|\nabla|_{1}} u(t)\right\|_{\dot{B}_{p, \infty}^{\sigma+2 \beta}} \lesssim \operatorname{esssup}_{0<t<T} t^{\frac{\beta}{\alpha}}\left\|\mathrm{e}^{\frac{1}{2 \alpha}|\nabla|_{1}} f(t)\right\|_{\dot{B}_{p, \infty}^{\sigma+2 \beta+1-2 \alpha}}
$$

for $0<t<T \leq \infty$, provided that the right-hand side term is finite.

\section{WELL-POSEDNESS OF THE NONLINEAR PROBLEM}

The well-posedness of the nonlinear equations (1) can be obtained by combining the estimates of the linear Stokes equations derived in Theorem 1 and the Banach contraction mapping principle. Therefore it is necessary to examine the nonlinear term of (1) though the pointwise multiplication $f g$ of functions $f$ and $g$ in suitable homogeneous spaces in the form

$$
\|f g\|_{Y^{2-4 \alpha}} \lesssim\|f\|_{Y^{1-2 \alpha}}\|g\|_{Y^{1-2 \alpha}} .
$$

To do so, we need the following function multiplication estimate.

Lemma 6. Let $1<p<q<\infty, s+\frac{d}{p}>0$, an integer $k>s+2 \frac{d}{p}$ and $(k-1) p<q<\infty$. Then there holds the estimate

$$
\begin{aligned}
& \left\|\mathrm{e}^{t^{\frac{1}{2 \alpha}}|\nabla|_{1}} f g\right\|_{\dot{B}_{p, \infty}^{s+\frac{d}{p}}} \\
& \lesssim \quad \sum_{j=1}^{k-1}\left\|\mathrm{e}^{t \frac{1}{2 \alpha}|\nabla|_{1}} f\right\|_{\dot{B}_{p, \infty}^{\frac{j s}{k}+\frac{d}{p}}}\left\|\mathrm{e}^{t \frac{1}{2 \alpha}|\nabla|_{1}} g\right\|_{\dot{B}_{p, \infty} \frac{(k-j) s}{k}+\frac{d}{p}} \\
& +\left\|\mathrm{e}^{t \frac{1}{2 \alpha}|\nabla|_{1}} f\right\|_{\dot{B}_{p, \infty}^{\frac{d}{p}-\frac{d}{q}}}\left\|\mathrm{e}^{t \frac{1}{2 \alpha}|\nabla|_{1}} g\right\|_{\dot{B}_{p, \infty}^{s+\frac{d}{p}+\frac{d}{q}}}+\left\|\mathrm{e}^{\frac{1}{2 \alpha}|\nabla|_{1}} g\right\|_{\dot{B}_{p, \infty}^{\frac{d}{p}-\frac{d}{q}}}\left\|\mathrm{e}^{t \frac{1}{2 \alpha}|\nabla|_{1}} f\right\|_{\dot{B}_{p, \infty}^{s+\frac{d}{p}+\frac{d}{q}}}
\end{aligned}
$$

for functions $f$ and $g$ on $R^{d}$, provided the right-hand side terms are finite. 
Proof. Let us begin with the proof of the inequality

$$
\|f g\|_{\dot{B}_{p, \infty}^{s+\frac{d}{p}}} \lesssim \sum_{j=1}^{k-1}\|f\|_{\dot{B}_{p, \infty}^{\frac{j s}{k}+\frac{d}{p}}}\|g\|_{\dot{B}_{p, \infty}^{\frac{(k-j) s}{k}+\frac{d}{p}}}+\|f\|_{\dot{B}_{p, \infty}^{\frac{d}{p}-\frac{d}{q}}}\|g\|_{\dot{B}_{p, \infty}^{s+\frac{d}{p}+\frac{d}{q}}}+\|g\|_{\dot{B}_{p, \infty}^{\frac{d}{p}-\frac{d}{q}}}\|f\|_{\dot{B}_{p, \infty}^{s+\frac{d}{p}+\frac{d}{q}}} \text {. }
$$

For the integer $k \geq s+\frac{d}{p}+\frac{d}{q}$, it is readily seen that the function product under the difference operator is subject to the binomial formula

$$
\triangle_{h}^{k}(f(x) g(x))=\sum_{j=0}^{k}\left(\begin{array}{c}
k \\
j
\end{array}\right) \triangle_{h}^{j} f(x) \triangle_{h}^{k-j} g(x+j h) .
$$

For the real number $r$ so that $\frac{1}{r}=\frac{1}{p}+\frac{1}{q}$, the selection of $q$ and $k$ implies that

$$
\frac{1}{r}<\frac{1}{p}+\frac{1}{(k-1) p} \text { or } \frac{k r}{k-1}>p .
$$

By the imbedding property (25), the characterization property (8) and the Hölder inequality, we find that

$$
\begin{aligned}
\|f g\|_{\dot{B}_{p, \infty}^{s+\frac{d}{p}}} & \lesssim\|f g\|_{\dot{B}_{r, \infty}^{s+\frac{d}{r}}} \\
& \lesssim \operatorname{essup}_{0 \neq h \in R^{d}} \frac{\left\|\sum_{j=0}^{k}\left(\begin{array}{c}
k \\
j
\end{array}\right) \triangle_{h}^{j} f \triangle_{h}^{k-j} g(\cdot+j h)\right\|_{L_{r}}}{|h|^{s+\frac{d}{r}}} \\
& \lesssim \operatorname{ess~sup}_{0 \neq h \in R^{d}} \frac{\sum_{j=1}^{k-1}\left\|\triangle_{h}^{j} f\right\|_{L_{\frac{k r}{j}}}\left\|\triangle_{h}^{k-j} g\right\|_{L_{\frac{k r}{k-j}}}+\|f\|_{L_{q}}\left\|\triangle_{h}^{k} g\right\|_{L_{p}}+\|g\|_{L_{q}}\left\|\triangle_{h}^{k} f\right\|_{L_{p}}}{|h|^{s+\frac{d}{r}}} \\
& \lesssim \sum_{j=1}^{k-1}\|f\|_{\dot{B}_{\frac{k r}{j}, \infty}^{\frac{j}{k}\left(s+\frac{d}{r}\right)}}\|g\|_{\dot{B}_{\frac{k r}{k-j}, \infty}^{\frac{(k-j)}{k}\left(s+\frac{d}{r}\right)}}+\|f\|_{L_{q}}\|g\|_{\dot{B}_{p, \infty}^{s+\frac{d}{r}}}+\|g\|_{L_{q}}\|f\|_{\dot{B}_{p, \infty}^{s+\frac{d}{r}}} .
\end{aligned}
$$

Since $\frac{k r}{j} \geq \frac{k r}{k-1}>p$, the imbedding property (25) implies that

$$
L_{q}\left(R^{d}\right) \hookrightarrow \dot{B}_{q, \infty}^{0}\left(R^{d}\right) \hookrightarrow \dot{B}_{p, \infty}^{\frac{d}{p}-\frac{d}{q}}\left(R^{d}\right) \text { and } \dot{B}_{\frac{k r}{j}, \infty}^{\frac{j}{k}\left(s+\frac{d}{r}\right)}\left(R^{d}\right) \hookrightarrow \dot{B}_{p, \infty}^{\frac{j}{k} s+\frac{d}{p}}\left(R^{d}\right) .
$$

Thus equation (30) becomes (29).

Since $\mathrm{e}^{t^{\frac{1}{2 \alpha}}|\nabla|_{1}} \triangle_{h}^{j} f=\triangle_{h}^{j} \mathrm{e}^{\frac{1}{2 \alpha}}|\nabla|_{1} f$, following the derivation of (29) by applying the inequality (12) instead of the Hölder inequality, we obtain (28) and hence complete the proof of Lemma 6.

Now we consider the nonlinear system described by (1). Let $u$ solve equation (1) associated with the initial condition

$$
u(0)=u_{0} \text { on } R^{d} .
$$

Taking the integral form of this system, we have the mild solution of equations (1) and (31) expressed as

$$
u(t)=\mathrm{e}^{-t(-\Delta)^{\alpha}} u_{0}+\left(\partial_{t}+(-\Delta)^{\alpha}\right)^{-1} P u \cdot \nabla u(t) .
$$

The well-posed result for equations (1) and (31) is stated in the following.

Theorem 2. Let $d \geq 2, \alpha>\frac{1}{2}, 1<p<\infty, 2-2 \alpha+\frac{d}{p}>0$ and $u_{0} \in \dot{B}_{p, \infty}^{1-2 \alpha+\frac{d}{p}}\left(R^{d}\right)$. Then there exists a constant $T>0$ such that equation (32) admits a unique solution $u \in$ $L_{\infty}\left(0, T ; \dot{B}_{p, \infty}^{1-2 \alpha+\frac{d}{p}}\left(R^{d}\right)\right)$ subject to the estimate

$$
\underset{0<t<T}{\operatorname{ess} \sup }\left(t^{\frac{\beta}{\alpha}}\left\|\mathrm{e}^{t^{\frac{1}{2 \alpha}}|\nabla|_{1}} u(t)\right\|_{\dot{B}_{p, \infty}^{2 \beta+1-2 \alpha+\frac{d}{p}}}+\left\|\mathrm{e}^{t^{\frac{1}{2 \alpha}}|\nabla|_{1}} u(t)\right\|_{\dot{B}_{p, \infty}^{1-2 \alpha+\frac{d}{p}}}\right) \lesssim\left\|u_{0}\right\|_{\dot{B}_{p, \infty}^{1-2 \alpha+\frac{d}{p}}}+1,
$$


where $\beta$ is a constant such that

$$
\alpha>\beta>0,2 \beta+1-2 \alpha>0,2 \beta+2-4 \alpha+\frac{d}{p}>0 .
$$

Additionally, if $u_{0}$ is sufficiently small in the space $\dot{B}_{p, \infty}^{1-2 \alpha+\frac{d}{p}}\left(R^{d}\right)$, the solution $u$ exists globally in time and the bound (33) remains true for $T \rightarrow \infty$.

Proof. For a constant $T>0$, we define the operator

$$
M_{u_{0}} u(t)=\mathrm{e}^{-t(-\Delta)^{\alpha}} u_{0}+\left(\partial_{t}+(-\Delta)^{\alpha}\right)^{-1} u(t)
$$

and the space

$$
X_{T}=\left\{u \in L_{\infty}\left(0, T ; \dot{B}_{p, \infty}^{1-2 \alpha+\frac{d}{p}}\left(R^{d}\right)\right) ;\|u\|_{X_{T}} \leq C\left\|u_{0}\right\|_{\dot{B}_{p, \infty}^{1-2 \alpha+\frac{d}{p}}}\right\}
$$

for a suitable constant $C$ and the norm

$$
\|u\|_{X_{T}}=\operatorname{esssup}_{0<t<T}\left(t^{\frac{\beta}{\alpha}}\left\|\mathrm{e}^{t^{\frac{1}{2 \alpha}}|\nabla|_{1}} u(t)\right\|_{\dot{B}_{p, \infty}^{2 \beta+1-2 \alpha+\frac{d}{p}}}+\left\|\mathrm{e}^{\frac{1}{2 \alpha}|\nabla|_{1}} u(t)\right\|_{\dot{B}_{p, \infty}^{1-2 \alpha+\frac{d}{p}}}\right) .
$$

It suffices to show that $M_{u_{0}}$ is a contraction operator mapping $X_{T}$ into itself.

Indeed, it is readily seen that

$$
\left\|\mathrm{e}^{t^{\frac{1}{2 \alpha}}|\nabla|_{1}} \mathrm{e}^{-t(-\Delta)^{\alpha}} u_{0}\right\|_{X_{T}} \lesssim\left\|u_{0}\right\|_{\dot{B}_{p, \infty}^{1-2 \alpha+\frac{d}{p}}}
$$

by taking into account the inequality (23) and the fact that $\mathrm{e}^{t^{\frac{1}{2 \alpha}}|\nabla|_{1}} \mathrm{e}^{-\frac{3}{4} t(-\Delta)^{\alpha}}$ is a bounded multiplier in $L_{p}\left(R^{d}\right)$ and hence in $\dot{B}_{p, \infty}^{1-2 \alpha+\frac{d}{p}}\left(R^{d}\right)$.

Let $u, v \in X_{T}$ and let the integer $k$ and the real number $q>p$ be given in Lemma 6 . It follows from Theorem 1 with $\sigma=1-2 \alpha+\frac{d}{p}$ and the inequality (28) with $s=2 \beta+2-4 \alpha$ that

$$
\begin{aligned}
& \left\|M_{u_{0}} u\right\|_{X_{T}} \lesssim\left\|u_{0}\right\|_{\dot{B}_{p, \infty}^{1-2 \alpha+\frac{d}{p}}}+\operatorname{esssup}_{t>0} t^{\frac{\beta}{\alpha}}\left\|\mathrm{e}^{\frac{1}{2 \alpha}|\nabla|_{1}} u u(t)\right\|_{\dot{B}_{p, \infty}^{2 \beta+2-4 \alpha+\frac{d}{p}}} \\
& \lesssim \quad\left\|u_{0}\right\|_{\dot{B}_{p, \infty}^{1-2 \alpha+\frac{d}{p}}}+\operatorname{ess}_{t>0} t^{\frac{\beta}{\alpha}}\left\|\mathrm{e}^{t^{\frac{1}{2 \alpha}}|\nabla|_{1}} u\right\|_{\dot{B}_{p, \infty}^{\frac{d}{p}-\frac{d}{q}}}\left\|\mathrm{e}^{t^{\frac{1}{2 \alpha}}|\nabla|_{1}} u\right\|_{\dot{B}_{p, \infty}^{2 \beta+2-4 \alpha+\frac{d}{p}+\frac{d}{q}}}
\end{aligned}
$$

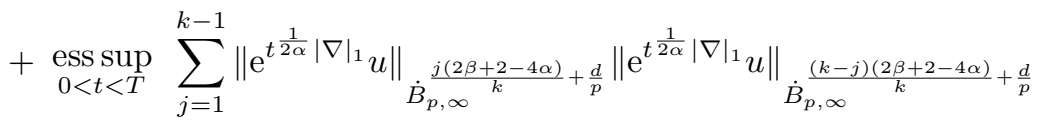

and

$$
\begin{aligned}
& \left\|M_{u_{0}} u-M_{u_{0}} v\right\|_{X_{T}} \\
& \lesssim \quad \operatorname{essiup}_{0<t<T} t^{\frac{\beta}{\alpha}}\left(\left\|\mathrm{e}^{t \frac{1}{2 \alpha}}|\nabla|_{1}(u-v) u\right\|_{\dot{B}_{p, \infty}^{2 \beta+2-4 \alpha+\frac{d}{p}}}+\left\|\mathrm{e}^{\frac{1}{t^{2 \alpha}}|\nabla|_{1}}(u-v) v\right\|_{\dot{B}_{p, \infty}^{2 \beta+2-4 \alpha+\frac{d}{p}}}\right) \\
& \lesssim \quad \operatorname{esssup}_{0<t<T} t^{\frac{\beta}{\alpha}}\left\|\mathrm{e}^{t^{\frac{1}{2 \alpha}}|\nabla|_{1}}(u-v)\right\|_{\dot{B}_{p, \infty}^{\frac{d}{p}-\frac{d}{q}}}\left\|\mathrm{e}^{t^{\frac{1}{2 \alpha}}|\nabla|_{1}} u\right\|_{\dot{B}_{p, \infty}^{2 \beta+2-4 \alpha+\frac{d}{p}+\frac{d}{q}}} \\
& +\underset{0<t<T}{\operatorname{esssup}} t^{\frac{\beta}{\alpha}}\left\|\mathrm{e}^{t \frac{1}{2 \alpha}|\nabla|_{1}} v\right\|_{\dot{B}_{p, \infty}^{\frac{d}{p}}-\frac{d}{q}}\left\|\mathrm{e}^{t \frac{1}{2 \alpha}|\nabla|_{1}}(u-v)\right\|_{\dot{B}_{p, \infty}^{2 \beta+2-4 \alpha+\frac{d}{p}+\frac{d}{q}}} \\
& +\operatorname{ess~sup}_{0<t<T} t^{\frac{\beta}{\alpha}} \sum_{j=1}^{k-1}\left\|\mathrm{e}^{t^{\frac{1}{2 \alpha}}|\nabla|_{1}}(u-v)\right\|_{\dot{B}_{p, \infty} \frac{j(2 \beta+2-4 \alpha)}{k}+\frac{d}{p}}\left\|\mathrm{e}^{\frac{1}{2 \alpha}|\nabla|_{1}} u\right\|_{\dot{B}_{p, \infty}} \frac{(k-j)(2 \beta+2-4 \alpha)}{k}+\frac{d}{p} \\
& +\operatorname{esssup}_{0<t<T} t^{\frac{\beta}{\alpha}} \sum_{j=1}^{k-1}\left\|\mathrm{e}^{\frac{1}{2 \alpha}|\nabla|_{1}}(u-v)\right\|_{\dot{B}_{p, \infty} \frac{j(2 \beta+2-4 \alpha)}{k}+\frac{d}{p}}\left\|\mathrm{e}^{t^{\frac{1}{2 \alpha}}|\nabla|_{1}} v\right\|_{\dot{B}_{p, \infty} \frac{(k-j)(2 \beta+2-4 \alpha)}{k}+\frac{d}{p}} .
\end{aligned}
$$

The number $q>p$ is selected to be sufficiently large so that $-2 \beta<1-2 \alpha+\frac{d}{q}<0$. Moreover we may assume the integer $k \geq 2$ so that

$$
1-\alpha<\frac{j}{k}(2 \beta+2-4 \alpha)<2 \beta+1-2 \alpha \text { for } j=1, \ldots, k .
$$


By Lemma 2, we have the real interpolation relations

$$
\begin{aligned}
& \dot{B}_{p, \infty}^{2 \beta+2-4 \alpha+\frac{d}{p}+\frac{d}{q}}\left(R^{d}\right)=\left(\dot{B}_{p, \infty}^{1-2 \alpha+\frac{d}{p}}\left(R^{d}\right), \dot{B}_{p, \infty}^{2 \beta+1-2 \alpha+\frac{d}{p}}\left(R^{d}\right)\right)_{1-\theta_{0}, \infty}, \\
& \dot{B}_{p, \infty}^{\frac{d}{p}-\frac{d}{q}}\left(R^{d}\right)=\left(\dot{B}_{p, \infty}^{1-2 \alpha+\frac{d}{p}}\left(R^{d}\right), \dot{B}_{p, \infty}^{2 \beta+1-2 \alpha+\frac{d}{p}}\left(R^{d}\right)\right)_{\theta_{0}, \infty}, \\
& \dot{B}_{p, \infty}^{\frac{(k-j)(2 \beta+2-4 \alpha)}{k}+\frac{d}{p}}\left(R^{d}\right)=\left(\dot{B}_{p, \infty}^{1-2 \alpha+\frac{d}{p}}\left(R^{d}\right), \dot{B}_{p, \infty}^{2 \beta+1-2 \alpha+\frac{d}{p}}\left(R^{d}\right)\right)_{1-\theta_{j}, \infty}, \\
& \dot{B}_{p, \infty}^{\frac{j(2 \beta+2-4 \alpha)}{k}+\frac{d}{p}}\left(R^{d}\right)=\left(\dot{B}_{p, \infty}^{1-2 \alpha+\frac{d}{p}}\left(R^{d}\right), \dot{B}_{p, \infty}^{2 \beta+1-2 \alpha+\frac{d}{p}}\left(R^{d}\right)\right)_{\theta_{j}, \infty}
\end{aligned}
$$

for

$$
\theta_{0}=\frac{2 \alpha-1-\frac{d}{q}}{2 \beta}, \theta_{j}=\frac{\frac{j}{k}(2 \beta+2-4 \alpha)-1+2 \alpha}{2 \beta}, j=1, \ldots, k-1 .
$$

We thus have

$$
\begin{aligned}
& \left\|M_{u_{0}} u\right\|_{X_{T}} \lesssim\left\|u_{0}\right\|_{\dot{B}_{p, \infty}^{1-2 \alpha+\frac{d}{p}}}+\operatorname{esssup}_{0<t<T} t^{\frac{\beta}{\alpha}}\left\|\mathrm{e}^{t^{\frac{1}{2 \alpha}}|\nabla|_{1}} u(t)\right\|_{\dot{B}_{p, \infty}^{2 \beta+1-2 \alpha+\frac{d}{p}}}\left\|\mathrm{e}^{t^{\frac{1}{2 \alpha}}|\nabla|_{1}} u(t)\right\|_{\dot{B}_{p, \infty}^{1-2 \alpha+\frac{d}{p}}} \\
& \lesssim \quad\left\|u_{0}\right\|_{\dot{B}_{p, \infty}^{1-2 \alpha+\frac{d}{p}}}+\underset{0<t<T}{\operatorname{ess} \sup } t^{\frac{\beta}{\alpha}}\left\|\mathrm{e}^{\frac{1}{2 \alpha}|\nabla|_{1}} u(t)\right\|_{\dot{B}_{p, \infty}^{2 \beta+1-2 \alpha+\frac{d}{p}}}\|u\|_{X_{T}}
\end{aligned}
$$

and

$$
\begin{aligned}
& \left\|M_{u_{0}} u-M_{u_{0}} v\right\|_{X_{T}} \\
& \lesssim \sum_{j=0}^{k-1}\left(\operatorname{ess~sup}_{0<t<T} t^{\frac{\beta}{\alpha}}\left\|\mathrm{e}^{t \frac{1}{2 \alpha}|\nabla|_{1}} u(t)\right\|_{\dot{B}_{p, \infty}^{2 \beta+1-2 \alpha+\frac{d}{p}}}\right)^{1-\theta_{j}}\|u\|_{X_{T}}^{\theta_{j}}\|u-v\|_{X_{T}} \\
& +\sum_{j=0}^{k-1}\left(\operatorname{ess}_{0<t<T} t^{\frac{\beta}{\alpha}}\left\|\mathrm{e}^{t \frac{1}{2 \alpha}|\nabla|_{1}} v(t)\right\|_{\dot{B}_{p, \infty}^{2 \beta+1-2 \alpha+\frac{d}{p}}}\right)^{\theta_{j}}\|v\|_{X_{T}}^{1-\theta_{j}}\|u-v\|_{X_{T}} .
\end{aligned}
$$

Since

$$
\lim _{t \rightarrow 0} t^{\frac{\beta}{\alpha}}\left\|\mathrm{e}^{\frac{1}{2 \alpha}}|\nabla|_{1} u(t)\right\|_{\dot{B}_{p, \infty}^{2 \beta+1-2 \alpha+\frac{d}{p}}}+\lim _{t \rightarrow 0} t^{\frac{\beta}{\alpha}}\left\|\mathrm{e}^{t^{\frac{1}{2 \alpha}}|\nabla|_{1}} v(t)\right\|_{\dot{B}_{p, \infty}^{2 \beta+1-2 \alpha+\frac{d}{p}}}=0,
$$

the estimates

$$
\left\|M_{u_{0}} u\right\|_{X_{T}} \leq C\left\|u_{0}\right\|_{\dot{B}_{p, \infty}^{1-2 \alpha+\frac{d}{p}}}
$$

and

$$
\left\|M_{u_{0}} u-M_{u_{0}} v\right\|_{X_{T}} \leq \frac{1}{2}\|u-v\|_{X_{T}}
$$

hold true for a small constant $T>0$ and a suitable constant $C$. Hence there exists a unique solution $u \in X_{T}$ satisfying $u=M_{u_{0}} u$ due to the Banach contraction principle.

For the existence of small global solution with $T \rightarrow \infty$, we adopt the space

$$
X_{\infty}=\left\{u \in L_{\infty}\left(0, \infty ; \dot{B}_{p, \infty}^{1-2 \alpha+\frac{d}{p}}\left(R^{d}\right)\right) ;\|u\|_{X_{\infty}} \leq\left\|u_{0}\right\|_{\dot{B}_{p, \infty}^{1-2 \alpha+\frac{d}{p}}}^{\frac{1}{2}}\right\}
$$

for $\|u\|_{X_{\infty}}$ expressed in (35) as $T \rightarrow \infty$. By (40) and (41), we have the following bounds

$$
\begin{array}{r}
\left\|M_{u_{0}} u\right\|_{X_{\infty}} \lesssim\left\|u_{0}\right\|_{\dot{B}_{p, \infty}^{1-2 \alpha+\frac{d}{p}}}+\|u\|_{X_{\infty}}^{2} \leq\left\|u_{0}\right\|_{\dot{B}_{p, \infty}^{1-2 \alpha+\frac{d}{p}}}^{\frac{1}{2}}, u \in X_{\infty}, \\
\left\|M_{u_{0}} u-M_{u_{0}} v\right\|_{X_{\infty}} \leq \frac{1}{2}\|u-v\|_{X_{\infty}}, u, v \in X_{\infty}
\end{array}
$$

provided that $\left\|u_{0}\right\|_{\dot{B}_{p, \infty}^{1-2 \alpha+\frac{d}{p}}}$ is sufficiently small. Therefore the global solution exists due to Banach contraction principle. The proof of Theorem 2 is complete. 
Remark 1. Note that $S\left(R^{d}\right)$ is not dense in $L_{\infty}\left(R^{d}\right)$ and hence is not dense in $\dot{B}_{p, \infty}^{1-2 \alpha+\frac{d}{p}}\left(R^{d}\right)$. That is,

$$
\left\|\mathrm{e}^{-t(-\Delta)^{\alpha}} u_{0}-u_{0}\right\|_{\dot{B}_{p, \infty}^{1-2 \alpha+\frac{d}{p}}} \not \rightarrow 0 \text { as } t \rightarrow 0
$$

for some $u_{0} \in \dot{B}_{p, \infty}^{1-2 \alpha+\frac{d}{p}}\left(R^{d}\right)$. Thus the continuity of the solution in the space $\dot{B}_{p, \infty}^{1-2 \alpha+\frac{d}{p}}\left(R^{d}\right)$ is generally not obtainable. However, if we define the space $\stackrel{\circ}{B}_{p, \infty}^{1-2 \alpha+\frac{d}{p}}\left(R^{d}\right) \subset \dot{B}_{p, \infty}^{1-2 \alpha+\frac{d}{p}}\left(R^{d}\right)$ to be the closure of $S\left(R^{d}\right)$ under the norm of $\dot{B}_{p, \infty}^{1-2 \alpha+\frac{d}{p}}\left(R^{d}\right)$, then the solution u given in Theorem 2 satisfies the continuity property

$$
u \in C\left([0, T) ; \stackrel{\circ}{B}_{p, \infty}^{1-2 \alpha+\frac{d}{p}}\left(R^{d}\right)\right) \text { whenever } u_{0} \in \stackrel{\circ}{B}_{p, \infty}^{1-2 \alpha+\frac{d}{p}}\left(R^{d}\right) .
$$

and hence solves the initial value problem defined by (1) and (31).

To show the continuity, we see that the proof of Theorem 1 yields the estimate

$$
\begin{aligned}
& \left\|\left(\partial_{t}+(-\Delta)^{\alpha}\right)^{-1} P u \cdot \nabla u(t)\right\|_{\dot{B}_{p, \infty}^{\sigma}}+t^{\frac{\beta}{\alpha}}\left\|\left(\partial_{t}+(-\Delta)^{\alpha}\right)^{-1} P u \cdot \nabla u(t)\right\|_{\dot{B}_{p, \infty}^{\sigma+2 \beta}} \\
& \quad \underset{0<t<T}{\operatorname{ess~sup}} t^{\frac{\beta}{\alpha}}\|u u\|_{\dot{B}_{p, \infty}^{\sigma+2 \beta+1-2 \alpha} .}
\end{aligned}
$$

This together with the proof of (40) using (29) instead of (28) implies that

$$
\begin{aligned}
& \left\|u(t)-u_{0}\right\|_{\dot{B}_{p, \infty}^{1-2 \alpha+\frac{d}{p}}} \\
& \quad \lesssim\left\|\mathrm{e}^{-t(-\Delta)^{\alpha}} u_{0}-u_{0}\right\|_{\dot{B}_{p, \infty}^{1-2 \alpha+\frac{d}{p}}}+\underset{0<t<T}{\operatorname{ess} \sup } t^{\frac{\beta}{\alpha}}\|u(t)\|_{\dot{B}_{p, \infty}^{2 \beta+1-2 \alpha+\frac{d}{p}}}\|u(t)\|_{\dot{B}_{p, \infty}^{1-2 \alpha+\frac{d}{p}}}
\end{aligned}
$$

for $0<t<T$. Hence we have

$$
\lim _{t \rightarrow 0}\left\|u(t)-u_{0}\right\|_{\dot{B}_{p, \infty}^{1-2 \alpha+\frac{d}{p}}} \lesssim \lim _{t \rightarrow 0}\left\|\mathrm{e}^{-t(-\Delta)^{\alpha}} u_{0}-u_{0}\right\|_{\dot{B}_{p, \infty}^{1-2 \alpha+\frac{d}{p}}}
$$

Moreover, for small $\epsilon>0$, the definition of the space $\stackrel{\circ}{B}_{p, \infty}^{1-2 \alpha+\frac{d}{p}}\left(R^{d}\right)$ ensures the existence of $v_{0} \in B_{p, \infty}^{1+\frac{d}{p}}\left(R^{d}\right)$ satisfying

This yields that

$$
\left\|u_{0}-v_{0}\right\|_{\dot{B}_{p, \infty}^{1-2 \alpha+\frac{d}{p}}}<\epsilon .
$$

$$
\begin{aligned}
& \left\|\mathrm{e}^{-t(-\Delta)^{\alpha}} u_{0}-u_{0}\right\|_{\dot{B}_{p, \infty}^{1-2 \alpha+\frac{d}{p}}} \\
& \quad \lesssim \quad\left\|u_{0}-v_{0}\right\|_{\dot{B}_{p, \infty}^{1-2 \alpha+\frac{d}{p}}}+\left\|\mathrm{e}^{-t(-\Delta)^{\alpha}}\left(u_{0}-v_{0}\right)\right\|_{\dot{B}_{p, \infty}^{1-2 \alpha+\frac{d}{p}}}+\left\|\mathrm{e}^{-t(-\Delta)^{\alpha}} v_{0}-v_{0}\right\|_{\dot{B}_{p, \infty}^{1-2 \alpha+\frac{d}{p}}} \\
& \quad \lesssim \quad \epsilon+\left\|u_{0}-v_{0}\right\|_{\dot{B}_{p, \infty}^{1-2 \alpha+\frac{d}{p}}}+\int_{0}^{t}\left\|(-\Delta)^{\alpha} \mathrm{e}^{-s(-\Delta)^{\alpha}} v_{0}\right\|_{\dot{B}_{p, \infty}^{1-2 \alpha+\frac{d}{p}}} d s \\
& \quad \lesssim \quad \epsilon+t\left\|v_{0}\right\|_{\dot{B}_{p, \infty}^{1+\frac{d}{p}}} .
\end{aligned}
$$

The combination of the previous equation and (42) gives the estimate

$$
\lim _{t \rightarrow 0}\left\|u(t)-u_{0}\right\|_{\dot{B}_{p, \infty}^{1-2 \alpha+\frac{d}{p}}} \lesssim \epsilon
$$

for any $\epsilon>0$. We thus obtain the continuity of the solution $u$ at the initial state $t=0$ and therefore the continuity of $u$ for $t>0$ due to the solution semigroup property of (1).

The Gevrey regularity result for $\alpha>\frac{1}{2}$ obtained in Theorem 2 is not extendable to the critical state $\alpha=\frac{1}{2}$ as the proof of generalized Hölder estimate (12) is derived from $L_{p}$ estimate, which is only available for $1<p<\infty$. When $\alpha=\frac{1}{2}$, it is necessary to consider the solution in the $L_{\infty}\left(R^{d}\right)$ space. Therefore we provide an application of Theorem 1 for $\alpha=\frac{1}{2}$ without involvement of Gevrey regularity. 
Theorem 3. Let $\alpha=\frac{1}{2}$ and $1<p<\infty$. Then there exists a constant $T>0$ such that initial value problem defined by (1) and (31) admits a unique solution $u \in C\left([0, T) ; \stackrel{\circ}{B}_{p, \infty}^{\frac{d}{p}}\left(R^{d}\right)\right)$ subject to the estimate

$$
\underset{0<t<T}{\operatorname{ess} \sup }\left(t^{2 \beta}\|u(t)\|_{\dot{B}_{p, \infty}^{2 \beta+\frac{d}{p}}}+\|u(t)\|_{\dot{B}_{p, \infty}^{\frac{d}{p}}}+\|u(t)\|_{L_{\infty}}\right) \lesssim\left\|u_{0}\right\|_{\dot{B}_{p, \infty}^{\frac{d}{p}}}+\left\|u_{0}\right\|_{L_{\infty}}
$$

for $0<\beta<\frac{1}{2}$, provided that the initial function $u_{0} \in \stackrel{\circ}{B^{\frac{d}{p}}}, \infty\left(R^{d}\right) \cap L_{\infty}\left(R^{d}\right)$.

Proof. The proof of Theorem 1 shows that

$$
\begin{aligned}
\|\left(\partial_{t}\right. & \left.+(-\Delta)^{\alpha}\right)^{-1} P u \cdot \nabla u(t)\left\|_{\dot{B}_{p, \infty}^{\frac{d}{p}}}+t^{2 \beta}\right\|\left(\partial_{t}+(-\Delta)^{\alpha}\right)^{-1} P u \cdot \nabla u(t) \|_{\dot{B}_{p, \infty}^{2 \beta+\frac{d}{p}}} \\
& +\left\|\left(\partial_{t}+(-\Delta)^{\alpha}\right)^{-1} P u \cdot \nabla u(t)\right\|_{L_{\infty}} \lesssim \underset{0<t<T}{\operatorname{esssup}} t^{2 \beta}\|u u(t)\|_{\dot{B}_{p, \infty}^{2 \beta+\frac{d}{p}}} .
\end{aligned}
$$

Defining the space

$$
X_{T}=\left\{u \in C\left([0, T) ; \stackrel{\circ}{B_{p, \infty}^{p}}\left(R^{d}\right)\right) ;\|u\|_{X_{T}} \leq C\left\|u_{0}\right\|_{\dot{B}_{p, \infty}^{\frac{d}{p}}}+C\left\|u_{0}\right\|_{L_{\infty}}\right\}
$$

for a constant $C$ and the norm

$$
\|u\|_{X_{T}}=\operatorname{essiup}_{0<t<T}\left(t^{2 \beta}\|u(t)\|_{\dot{B}_{p, \infty}^{2 \beta+\frac{d}{p}}}+\|u(t)\|_{\dot{B}_{p, \infty}^{\frac{d}{p}}}+\|u(t)\|_{L_{\infty}}\right)
$$

and recalling $M_{u_{0}} u$ given in (34), we use equation (44) to obtain

$$
\left\|M_{u_{0}} u-\mathrm{e}^{-t(-\Delta)^{\alpha}} u_{0}\right\|_{X_{T}} \lesssim \quad \operatorname{esssup}_{0<t<T} t^{2 \beta}\|u u(t)\|_{\dot{B}_{p, \infty}^{2 \beta+\frac{d}{p}}} .
$$

To estimate the right-hand side term of this equation, we employ (30), (38) and (39) to obtain

$$
\begin{aligned}
\|u u\|_{\dot{B}_{p, \infty}^{2 \beta+\frac{d}{p}}} & \lesssim \sum_{j=1}^{k-1}\|u\|_{\dot{B}_{\frac{k p}{p}, \infty}^{\frac{j}{k}\left(2 \beta+\frac{d}{p}\right)}}\|u\|_{\dot{B}_{\frac{k p}{k-j}, \infty}^{\frac{k-j}{k}\left(2 \beta+\frac{d}{p}\right)}}+\|u\|_{L_{\infty}}\|u\|_{\dot{B}_{p, \infty}^{2 \beta+\frac{d}{p}}} \\
& \lesssim \sum_{j=1}^{k-1}\|u\|_{\dot{B}_{p, \infty}^{\frac{2 \beta j}{k}+\frac{d}{p}}}\|u\|_{\dot{B}_{p, \infty}^{\frac{2 \beta(k-j)}{k}+\frac{d}{p}}}+\|u\|_{L_{\infty}}\|u\|_{\dot{B}_{p, \infty}^{2 \beta+\frac{d}{p}}} \\
& \lesssim\|u\|_{\dot{B}_{p, \infty}^{\frac{d}{p}}}\|u\|_{\dot{B}_{p, \infty}^{2 \beta+\frac{d}{p}}}+\|u\|_{L_{\infty}}\|u\|_{\dot{B}_{p, \infty}^{2 \beta+\frac{d}{p}}}
\end{aligned}
$$

for an integer $k>2 \beta+\frac{d}{p}$. Therefore (45) becomes

$$
\left\|M_{u_{0}} u-\mathrm{e}^{-t(-\Delta)^{\alpha}} u_{0}\right\|_{X_{T}} \lesssim \quad \operatorname{esssup}_{0<t<T} t^{2 \beta}\|u(t)\|_{\dot{B}_{p, \infty}^{2 \beta+\frac{d}{p}}}\|u\|_{X_{T}} .
$$

This combined with Remark 1 gives the continuity

$$
\lim _{t \rightarrow 0}\left\|M_{u_{0}} u(t)-u_{0}\right\|_{\dot{B}_{p, \infty}^{\frac{d}{p}}} \lesssim \lim _{t \rightarrow 0}\left\|\mathrm{e}^{-t(-\Delta)^{\alpha}} u_{0}-u_{0}\right\|_{\dot{B}_{p, \infty}^{\frac{d}{p}}}=0
$$

and the boundedness

$$
\begin{aligned}
\left\|M_{u_{0}} u\right\|_{X_{T}} & \lesssim\left\|\mathrm{e}^{-t(-\Delta)^{\alpha}} u_{0}\right\|_{X_{T}}+\underset{0<t<T}{\operatorname{ess} \sup ^{2 \beta}\|u(t)\|_{\dot{B}_{p, \infty}^{2 \beta+\frac{d}{p}}}\|u\|_{X_{T}}} \\
& \leq C\left(\left\|u_{0}\right\|_{\dot{B}_{p, \infty}^{\frac{d}{p}}}+\left\|u_{0}\right\|_{L_{\infty}}\right)
\end{aligned}
$$

for a suitable choice of the constant $C$, since $\lim _{t \rightarrow 0} t^{2 \beta}\|u(t)\|_{\dot{B}_{p, \infty}^{2 \beta+\frac{d}{p}}}=0$. This shows that the operator $M_{u_{0}}$ maps the space $X_{T}$ into itself when $T>0$ is sufficiently small. Similarly, we also have the contraction property

$$
\left\|M_{u_{0}} u-M_{u_{0}} v\right\|_{X_{T}} \leq \frac{1}{2}\|u-v\|_{X_{T}} \text { for } u, v \in X_{T} .
$$


Since $X_{T}$ is a complete metric space, the operator $M_{u_{0}}$ has a unique fixed point in $X_{T}$ as a result of the Banach contraction principle. This completes the proof of Theorem 3 .

It should be mentioned that the well-posedness result described by Theorem 2 is essentially based Theorem 1 and Lemma 6 , by which the norm of the negative degree homogeneous space $\dot{B}_{p, \infty}^{\frac{d}{p}-\frac{d}{q}}\left(R^{d}\right)$ in Lemma 6 can be controlled by those of the positive degree homogeneous space $\dot{B}_{p, \infty}^{2 \beta+1-2 \alpha+\frac{d}{p}}\left(R^{d}\right)$ and the negative degree homogeneous space $\dot{B}_{p, \infty}^{1-2 \alpha+\frac{d}{p}}\left(R^{d}\right)$ in Theorem 1 for $\frac{1}{2}<\alpha$. For the weak dissipation situation $0<\alpha<\frac{1}{2}$, the negative degree homogeneous space $\dot{B}_{p, \infty}^{\frac{d}{p}-\frac{d}{q}}\left(R^{d}\right)$ is no longer an interpolation space between the two positive degree homogeneous spaces $\dot{B}_{p, \infty}^{2 \beta+1-2 \alpha+\frac{d}{p}}\left(R^{d}\right)$ and $\dot{B}_{p, \infty}^{1-2 \alpha+\frac{d}{p}}\left(R^{d}\right)$. Thus for controlling the nonlinear term of (1), the Hölder like estimate in Lemma 6 should be improved. To do so, it is useful to consider the Hölder like estimate involving derivatives as related to commutator estimates $[8,14,15,21]$.

Acknowledgment. The research is partially supported by the NSFC(grant No. 11571240) of China.

\section{References}

\section{REFERENCES}

[1] H. Bae, A. Biswas, Gevrey regularity for a class of dissipative equations with analytic nonlinearity, arxiv:math/1403.1603v1.

[2] A. Biswas, Gevrey regularity for the supercritical quasi-geostrophic equation, J Diff. Equs. 257 (2014) 1753-1772.

[3] M. Cannone, Harmonic Analysis tools for solving the incompressible Navier-Stokes equations, Handbook mathematical fluid dynamics, Chap. 3, 161-244, 2004.

[4] C. Cao, M. A. Rammaha, E. S. Titi, The Navier-Stokes equations on the rotating 2-D sphere: Gevrey regularity and asymptotic degrees of freedom, Z. Angew. Math. Phys. 50 (1999), 341-360.

[5] D. Chae, P. Constantin, J. Wu, Inviscid models generalizing the 2D Euler and the surface quasigeostrophic equations, Arch. Rat. Mech. Anal. 202 (2011), 35-62

[6] D. Chae, P. Constantin, J. Wu, Dissipative models generalizing the 2D Navier-Stokes and the surface quasi-geostrophic equations, Indiana Univ. Math. J. 61 (2012), 1997-2018.

[7] J. G. Charney, J. G. DeVore, Multiple flow equilibria in the atmosphere and blocking, J. Atmos. Sci. 36 (1979), 1205-1216.

[8] Q. Chen, C. Miao, Z. Zhang, A new Bernsteins inequality and the 2D dissipative quasi-geostrophic equation, Commun. Math. Phys. 271 (2007), 821-838.

[9] Z. -M. Chen, Steady-state bifurcation analysis of a strong nonlinear atmospheric vorticity equation, J. Math. Anal. Appl. 431 (2015), 1-21.

[10] Z. -M. Chen, Bifurcating steady-state solutions of the dissipative quasi-geostrophic equation in Lagrangian formulation, Nonlinearity 29 (2016), 3132-3147

[11] Z. -M. Chen, Z. Xin, Homogeneity criterion for the Navier-Stokes equations in the whole spaces, J. Math. Fluid Mech. 3 (2001), 152-182.

[12] Z. -M. Chen, X. Xiong, Equilibrium states of the Charney-DeVore quasi-geostrophic equation in midlatitude atmosphere, J. Math. Anal. Appl. 444 (2016), 1403-1416.

[13] P. Constantin, J. Wu, Behavior of solutions of 2D quasi-geostrophic equations, SIAM J. Math. Anal. 30 (1999), 937-948.

[14] R. Danchin, Global existence in critical spaces for compressible Navier-Stokes equations, Invent. Math. 141 (2000), 579-614.

[15] R. Danchin, Density-dependent incompressible viscous fluids in critical spaces. Proc. Roy. Soc. Edinburgh A 133 (2003), 1311-1334.

[16] H. Dong, Dissipative quasi-geostrophic equations in critical Sobolev spaces: smoothing effect and global well-posedness, Discrete Contin. Dyn. Syst. 26 (2010), 1197-1211.

[17] H. Dong, D. Li, Spatial analyticity of the solutions to the subcritical dissipative quasi-geostrophic equations, Arch. Rat. Mech. Anal. 189 (2008), 131-158.

[18] C. Foias, R. Temam, Gevrey class regularity for the solutions of the Navier-Stokes equations, J. Funct. Anal. 87 (1989), 359-369.

[19] T. Kato, On classical solutions of the two-dimensional non-stationary Euler equation, Arch. Rat. Mech. Anal. 25 (1967), 188-200. 
[20] T. Kato, Strong $L^{p}$-solutions of the Navier-Stokes equation in $R^{m}$, with applications to weak solutions, Math. Z. 187 (1984), 471-480.

[21] T. Kato, G. Ponce, Commutator estimates and the Euler and Navier-Stokes equations, Comm. Pure Appl. Math. XLI (1988), 891-907.

[22] N. H. Katz, N. Pavlovi, A cheap Caffarelli-Kohn-Nirenberg inequality for the Navier-Stokes equation with hyper-dissipation. Geom. Funct. Anal. (2002), 355-379.

[23] A. Kiselev, Some recent results on the critical surface quasi-geostrophic equation: a review. Hyperbolic problems: theory, numerics and applications. Proc. Sympos. Appl. Math., Vol. 67, Part 1. AMS, Providence, RI, 105-122, 2009

[24] A. Kiselev, F. Nazarov, A. Volberg, Global well-posedness for the critical 2D dissipative quasigeostrophic equation, Invent. Math. 167 (2007) 445-453.

[25] O. Ladyzhenskaya, The Mathematical Theory of Viscous Incompressible Flow, Gordon \& Breach, New York, 1969

[26] G. Lemarié-Rieusset, Recent Developments in the Navier-Stokes Problem, Chapman \& Hall/CRC, London, 2002.

[27] J. Leray, Sur le mouvement d'un liquide visqueux emplissant l'espace. Acta Math. 63 (1934), $193-248$.

[28] P. Li, Z. Zhai, Well-posedness and regularity of generalized Navier-Stokes equations in some critical Q-spaces, J. Funct. Anal. 259 (2010), 2457-2519.

[29] L. Nirenberg, On elliptic partial differential equations, Ann. Sc. Norm. Sup. Pisa 13 (1959) $123-131$.

[30] E. Stein, Singular Integrals and Differentiability Properties of Functions, Princeton University Press, Princeton, New Jersey, 1970.

[31] T. Tao, Global regularity for a logarithmically supercritical hyper-dissipative Navier- Stokes equation, Anal. PDE 2 (2009), 361-366.

[32] T. Tao, Finite time blowup for an averaged three-dimensional Navier-Stokes equation, J. Amer. Math. Soc. 29 (2016), 601-674.

[33] H. Triebel, Theory of Function Spaces, Birkhäuser Verlag, Basel,1983

[34] H. Triebel, Theory of Function Spaces II, Birkhäuser Verlag, Basel,1992

[35] G. Wolansky, Existence uniqueness and stability of stationary barotropic flow with forcing and dissipation, Comm. Pure Appl. Math. 41 (1988), 19-46.

[36] J. Wu, Quasi-geostrophic-type equations with initial data in Morrey spaces, Nonlinearity 10 (1997), 1409-1420.

[37] J. Wu, Generalized MHD equations, J. Differential Equations 195 (2003), 284-312.

[38] J. Wu, The generalized incompressible Navier-Stokes equations in Besov spaces, Dyn. Partial Diff. Equ. 1 (2004), 381-400

[39] J. Wu, Lower bounds for an integral involving fractional Laplacians and the generalized Navier-Stokes equations in Besov spaces, Commun. Math. Phys. 263 (2005), 803-831.

[40] J. Xiao, Homothetic variant of fractional Sobolev space with application to Navier-Stokes system, Dyn. Partial Diff. Equ. 2 (2007), 227-245.

[41] V. Yudovich, Nonstationary flow of an ideal incompressible liquid, Zhurn. Vych. Mat. 3 (1963), 10321066.

Ship Science, University of Southampton, Southampton SO16 7QF, UK

E-mail address: zhimin@soton.ac.uk

School of Mathematics and Statistics, Shenzhen University, Shenzhen 518052, China 